\title{
Hepatocellular Carcinoma: Current Concepts in Diagnosis, Staging and Treatment
}

\author{
Kerstin Schütte Christian Schulz Peter Malfertheiner \\ Department of Gastroenterology, Hepatology and Infectious Diseases, Otto von Guericke \\ University, Magdeburg, Germany
}

\section{Key Words}

Diagnosis $\cdot$ Hepatocellular carcinoma $\cdot$ Staging $\cdot$ Treatment

\begin{abstract}
Background: Hepatocellular carcinoma (HCC) significantly contributes to the global burden of cancer. Liver cancer is the third most frequent cause of cancer-related death, with HCC representing more than $90 \%$ of primary liver cancers. During the last decade, much progress has been made with respect to the definition of patient populations at risk who may benefit from surveillance strategies, as well as in the diagnosis and treatment of the disease.

Summary: New locoregional and systemic therapies have significantly increased the survival of patients with HCC. A multitude of clinical trials addressing patients with HCC have led to advancements in the allocation of subgroups of patients to their optimal individual treatment strategy. This review provides an overview on recent developments in diagnostic and therapeutic modalities and an outlook on future directions in the management of HCC.

Key Message: New locoregional and systemic therapies in patients with HCC have significantly improved clinical outlook and patient survival. This review provides an overview of recent developments in diagnostics and therapeutics, as well as an outlook on future directions in the management of HCC.

Practical Implications: Transabdominal ultrasound is the method of choice for the surveillance of patients at risk of developing HCC, and any suspicious focal lesion in the liver should be characterized. Contrast-enhanced ultrasound with application of second-generation contrast agents enables visualization of tumor vascularity. The Barcelona Clinic Liver Cancer $(B C L C)$ system is the most widely used classification system to guide the clinical management of HCC. Liver resection should be considered for patients with well-preserved liver function and without portal hypertension or elevated bilirubin. Patients not suitable for resection should undergo local tumor ablation. Candidates who are eligible for liver transplantation
\end{abstract}


may benefit from bridging therapy during their waiting period. Patients in the BCLC B category benefit from locoregional transarterial therapy; transarterial chemoembolization is the treatment of choice. Sorafenib is the only systemic therapy that has been shown to prolong overall survival in patients with advanced disease, and is therefore recommended in patients with well-preserved liver function.

(C) 2014 S. Karger AG, Basel

\section{Introduction}

Hepatocellular carcinoma (HCC) significantly contributes to the global burden of cancer. Liver cancer is the third most frequent cause of cancer-related death, with HCC representing more than $90 \%$ of primary liver cancers [1]. During the last decade much progress has been made with respect to definition of patient populations at risk who benefit from surveillance strategies as well as to diagnosis and treatment of the disease. This has led to the publication of several staging and treatment algorithms and not less than 17 guidelines worldwide on the management of HCC. Recent updates of the guidelines developed by the American Association for the Study of Liver Diseases (AASLD) as well as by the European Association for the Study of the Liver (EASL) have been released [2-4]. All guidelines provide a concise summary on current evidence-based approaches. This review gives a short overview on the major topics in the clinical management of HCC in combination with an outlook on possible future directions.

\section{Epidemiology}

Annually, more than 500,000 patients are diagnosed with liver cancer worldwide, with HCC accounting for $85-90 \%$ of all cases. The highest prevalence of HCC is in developing countries with more than $80 \%$ of cases. Both incidence and mortality rates are significantly higher in male subjects $[1,5]$. In parallel to the geographic variations in the distribution of HCC, a diversity in etiology and in the age at disease onset can be observed. Worldwide hepatitis $\mathrm{B}$ virus (HBV) infection is the most dominant risk factor and accounts for at least $50 \%$ of HCC cases, in spite of the fact that a vaccine against HBV has been available for more than 20 years. In regions with predominant vertical transmission of HBV (e.g. Eastern Asia), patients are diagnosed with HCC at a younger age in comparison to North America and Europe, where hepatitis $\mathrm{C}$ virus (HCV) infection is the leading viral cause of HCC. Because of the predominant horizontal transmission of HCV infection in adulthood, the onset of HCC occurs in more advanced age. Besides alcoholic liver cirrhosis, the metabolic syndrome (diabetes mellitus, obesity) has become more and more important as a causative factor during the last decades [1], especially in developed regions of the world, which may lead to a definition of a larger target population for surveillance in the future.

\section{Surveillance}

Transabdominal ultrasound is the method of first choice for the surveillance of patients at risk of developing HCC. Low costs, non-invasiveness, absence of procedure-related risks and wide availability in combination with a high diagnostic accuracy are the key features of the method. However, it should be emphasized that examiners need to be trained adequately with the method as the quality of the examination strongly depends on the experience of the 
examiner. Furthermore, the quality of the ultrasound machine and the physical condition of the patient are important factors. HCC is diagnosed by ultrasound with a sensitivity of $86 \%$ and a specificity of $77 \%$ [6]. Alternative imaging techniques for patients at risk are currently not recommended as surveillance tools because they are not cost-effective. Only in special settings, e.g. patients on the waiting list for liver transplantation or in case of insufficient assessment of the liver by ultrasound, a more sensitive and objective imaging method (CT or MRI) should be considered. Repeated examination at 6-month intervals is recommended according to published guidelines [2, 3, 7]. The use of alpha-fetoprotein (AFP) in the surveillance and diagnosis of HCC is no longer recommended in the current EASL and AASLD guidelines due to its low specificity, whereas it is part of the recommended surveillance in combination with ultrasound in the Asia Pacific region [7]. The diagnostic yield of tumor markers is enhanced by a combination of different biomarkers added to AFP. The combination of des-gamma-carboxy prothrombin (DCP) and AFP-L3\% with the assessment of AFP levels improved both sensitivity and specificity in the diagnosis of HCC $[8,9]$. Further effort needs to be invested for the development and implementation of a set of biomarkers into surveillance strategies of patients at risk.

\section{Diagnosis}

Suspicious focal lesions in the liver detected by transabdominal ultrasound need to be characterized. The distinction of HCC from focal liver lesions of other origin is mandatory.

Significant changes in the vascular supply of nodules occur during hepatocarcinogenesis that allow non-invasive differentiation of dysplastic nodules from HCC. In dysplastic nodules portal venous flow is maintained but hepatic arterial flow is decreased, whereas hepatic arterial flow increases in early HCC accompanied by a decrease in portal venous flow. In advanced HCC the blood supply is maintained by the abnormal hepatic artery branches. Tumor cells progressively replace normal bile canaliculi and normally functioning hepatocytes [10]. In the cirrhotic liver, the diagnosis of HCC can therefore be based on non-invasive criteria applying dynamic contrast-enhanced imaging modalities searching for the unique dynamic radiological features of HCC, with early contrast uptake in the arterial phase (hypervascularization) and washout in the portal venous or delayed phases. However, to detect the radiological hallmarks of HCC in a cirrhotic liver, special requirements on the quality of imaging have to be met. Diagnostic CT should comprise at least three phases following the injection of contrast media (arterial, portal-venous and late phase). Dynamic contrastenhanced MRI is the only alternative imaging method to CT accepted in Western guidelines.

Contrast-enhanced ultrasound (CEUS) with application of second-generation contrast agents such as SonoVue ${ }^{\circledR}$ allows excellent visualization of tumor vascularity and has been shown to be accurate in the diagnosis of HCC in multicenter studies. A recent meta-analysis reported a sensitivity of $81 \%$ and a specificity of $86 \%$ in HCC $<2 \mathrm{~cm}$ in size when compared to histology [11]. However, CEUS was not recommended as a diagnostic tool in the current AASLD and EASL guidelines because of low specificity with inadequate differentiation from intrahepatic cholangiocarcinoma [12] and the fact that CEUS is not available in the USA. The fact has raised controversial discussions [13] in Europe, and the recently published German S3 guidelines explicitly include CEUS as a first-line diagnostic option in the non-invasive diagnosis of HCC [14].

Specific new-generation contrast media offer the chance of further improvement in the diagnostic yield of MRI and ultrasound. However, the use of liver-specific contrast media in MRI imaging is not part of the current recommendations in the EASL and AASLD guidelines either. Gadoxetic acid, Gd-EOB-DTPA, is a hepatocyte-specific contrast agent for MRI that is taken up into the hepatocytes and secreted into the biliary system via distinct transporter 
Table 1. Performance of imaging modalities in the detection and characterization of HCC

\begin{tabular}{|c|c|c|c|c|c|c|}
\hline \multirow[t]{2}{*}{ Method } & $<1 \mathrm{~cm}, \%$ & \multicolumn{2}{|l|}{$1-2 \mathrm{~cm}, \%$} & $>2 \mathrm{~cm}, \%$ & \multicolumn{2}{|l|}{ Any size, $\%$} \\
\hline & sensitivity specificity & sensitivity & specificity & sensitivity specificity & sensitivity & specificity \\
\hline Ultrasound $[18,41]$ & & $21-51.7$ & 93 & & 46 & \\
\hline $\begin{array}{l}\text { Contrast-enhanced } \\
\text { ultrasound [42-45] }\end{array}$ & & $53-86.7$ & $91-100$ & & $79.3-86.1$ & $79.2-96.6$ \\
\hline CT $[41,42,46,47]$ & $14.3-21.4$ & $53-80$ & 99 & $91.8-93.9$ & $44-78.1$ & $96-100$ \\
\hline MRI $[41,42,46,47]$ & $42.9-64.3$ & $40-100$ & 100 & 98 & $44-91.8$ & $87-100$ \\
\hline $\begin{array}{l}\text { Gd-EOB-DTPA- } \\
\text { enhanced MRI }[46,48]\end{array}$ & $42.9-96$ & $80-100$ & & 98.7 & $86.3-98$ & 74.2 \\
\hline
\end{tabular}

proteins [10]. Assessment of the hepatobiliary phase in MRI after application of gadoxetic acid further enhances the sensitivity of MRI in the detection of HCC [15]. Sonazoid is a hepatocyte-specific ultrasound contrast agent that also results in a 'washout' of HCC in the postvascular Kupffer cell phase. In Asia it is therefore recommended as a decisional tool concerning hypovascular lesions $<1 \mathrm{~cm}$ in size [16]. Sonazoid-enhanced ultrasound was even more sensitive in the detection of focal liver lesions than CT [17].

In the non-cirrhotic liver and in case of atypical contrast behavior of the liver lesion in CEUS, CT or MRI histological diagnosis of HCC is demanded. However, the high incidence of sampling errors when performing biopsies on small focal liver lesions with false-negative findings in up to $30 \%$ of lesions $<2 \mathrm{~cm}$ [18] needs to be considered when comparing the performance of non-invasive methods to histology as the gold standard. As there is a risk of tumor seeding of $2.7-11 \%[19,20]$, fine needle biopsy should only be performed in wellselected cases and in case of clinical consequence of biopsy.

Tumor size is a critical factor impacting on the diagnostic accuracy of invasive and noninvasive methods in HCC (table 1). Algorithms for the non-invasive diagnosis of HCC in the cirrhotic liver therefore give recommendations depending on the size of the lesion (table 2). In case of a nodule $>1 \mathrm{~cm}$ in size, the AASLD proposes one high-quality imaging method with evidence of typical radiological hallmarks as being sufficient for the diagnosis of HCC. The European guidelines propose a more conservative approach for lesions 1-2 cm in size, with a second confirmatory imaging method recommended in non-excellence centers. In the Asia Pacific region the size of a lesion does not matter if it shows typical hallmarks in one contrastenhanced imaging.

\section{Staging}

Tumor stage, liver function and clinical performance status of the patient are the three major factors that influence therapeutic decisions and prognosis in patients with HCC. Several staging systems involving these factors have been developed for the specific assessment of patients with allocation to prognostic groups and treatment apart from the TNM system. The most disseminated and applied system directing the clinical management of HCC in the Western world is the Barcelona Clinic Liver Cancer (BCLC) classification [21] (table 3).

HCC with extranodular growth or of the confluent multinodular type more frequently presents with satellite lesions than early HCC and the single nodular type [22]. Poor differen- 
Table 2. Diagnostic algorithms with respect to size of lesion in the cirrhotic liver in different guidelines

\begin{tabular}{|c|c|c|c|}
\hline Guideline & $<1 \mathrm{~cm}$ & $1-2 \mathrm{~cm}$ & $>2 \mathrm{~cm}$ \\
\hline AASLD [2] & $\begin{array}{l}\text { Repeat ultrasound at } 3 \text { months, further } \\
\text { investigations in case of growth or } \\
\text { change in character. }\end{array}$ & \multicolumn{2}{|c|}{$\begin{array}{l}\text { Any lesion of } \geq 1 \mathrm{~cm} \text { in size: } 4 \text {-phase multidetector CT or } \\
\text { dynamic contrast MRI with arterial hypervascularity and } \\
\text { venous or delayed phase washout; otherwise biopsy. }\end{array}$} \\
\hline EASL [3] & $\begin{array}{l}\text { Repeat ultrasound at } 4 \text { months, further } \\
\text { investigations in case of growth. }\end{array}$ & $\begin{array}{l}\text { 4-phase CT/dynamic contrast- } \\
\text { enhanced MRI. One positive } \\
\text { modality showing hallmarks of HCC } \\
\text { sufficient in centers of excellence, } \\
\text { otherwise confirmation by second } \\
\text { modality recommended. Biopsy in } \\
\text { case of missing hallmarks. }\end{array}$ & $\begin{array}{l}\text { 4-phase CT or dynamic } \\
\text { contrast-enhanced } \\
\text { MRI with confirmation } \\
\text { of radiological } \\
\text { hallmarks in one } \\
\text { imaging technique, } \\
\text { otherwise biopsy. }\end{array}$ \\
\hline
\end{tabular}

APASL [7] Typical HCC can be diagnosed by imaging regardless of size if a typical vascular pattern, i.e. arterial enhancement with portal-venous washout, is obtained on dynamic CT, dynamic MRI or CEUS.

German S3 Arterial hypervascularization and rapid washout of contrast media with reversal of contrast in comparison guideline to surrounding liver parenchyma is sufficient in high-risk patients and securely detectable in tumors $>1 \mathrm{~cm}$. Three-phased contrast-enhanced cross-sectional imaging is necessary. CT, MRI and CEUS are options. Focal liver lesions $<1 \mathrm{~cm}$ should undergo follow-up with contrast-enhanced imaging in a 3-month interval. In case of missing hallmarks, biopsy is recommended for lesions $<2 \mathrm{~cm}$. In lesions $>2 \mathrm{~cm}$, a second contrastenhanced imaging method is recommended, but biopsy is also an option.

ESMO-ESDO No clear recommendations with respect to size of lesion. Multiple-phase CT or dynamic contrast-enhanced guideline [19] MRI with detection of typical vascular hallmark of HCC.

APASL = Asian Pacific Association for the Study of the Liver; ESMO-ESDO = European Society for Medical Oncology-European Society of Digestive Oncology.

Table 3. The BCLC staging system and treatment strategy (adopted from [3])

\begin{tabular}{|c|c|c|c|c|c|c|}
\hline Stage & Tumor extension & Liver function & $\begin{array}{l}\text { Clinical } \\
\text { PS }\end{array}$ & $\begin{array}{l}\text { Recommended } \\
\text { treatment }\end{array}$ & Target population & Prognosis \\
\hline 0 & single nodule $<2 \mathrm{~cm}$ & $\begin{array}{l}\text { Child-Pugh A, no } \\
\text { portal hypertension }\end{array}$ & 0 & resection & $\begin{array}{l}\text { curative intent: } \\
30-40 \% \text { of patients }\end{array}$ & $\begin{array}{l}\text { median OS } \\
>60 \text { months }\end{array}$ \\
\hline A & $\begin{array}{l}\text { single nodule } 2-5 \mathrm{~cm} \\
\text { or } 3 \text { nodules } \leq 3 \mathrm{~cm}\end{array}$ & Child-Pugh A or B & 0 & $\begin{array}{l}\text { liver transplantation, } \\
\text { RFA/PEI }\end{array}$ & $\begin{array}{l}\text { curative intent: } \\
30-40 \% \text { of patients }\end{array}$ & $\begin{array}{l}\text { median OS } \\
>60 \text { months }\end{array}$ \\
\hline $\mathrm{B}$ & multinodular & Child-Pugh A or B & 0 & TACE & $20 \%$ & OS 20 months \\
\hline $\mathrm{C}$ & $\begin{array}{l}\text { portal invasion or } \mathrm{N} 1 \\
\text { or } \mathrm{M} 1\end{array}$ & Child-Pugh A or B & $1-2$ & $\begin{array}{l}\text { systemic treatment } \\
\text { with sorafenib }\end{array}$ & $40 \%$ & OS 11 months \\
\hline $\mathrm{D}$ & any & Child C & $>2$ & best supportive care & $10 \%$ & OS $<3$ months \\
\hline
\end{tabular}

OS = Overall survival; PEI = percutaneous ethanol injection; PS = performance status.

tiation and a size $>5 \mathrm{~cm}$ are associated with increased risk for intrahepatic spread [23]. Data on well-evaluated cohorts of patients concerning the extrahepatic manifestations of HCC are still lacking, and knowledge concerning the incidence of metastases is derived from large case series and autopsy studies. The lungs (18-60\%), abdominal lymph nodes (27-42\%) and 
bones $(2-25 \%)[24,25]$ are the most frequent sites of distant metastasis in clinical series, while in an autopsy series the diaphragm and the adrenal glands were more frequent metastatic sites than the bones [26]. Younger age, high expression of AFP in the serum and vascular invasion are significant predictors of development of metastatic disease [24]. It is still debated whether the occurrence of extrahepatic spread significantly influences the survival of patients $[27,28]$ or whether intrahepatic progressive tumor disease is the most relevant factor in the presence of extrahepatic spread. Intrahepatic tumor burden assessment by contrast-enhanced MRI is recommended in the current guidelines and critical for decision-making in patients who are planned for liver resection or transplantation. CT provides optimal information in the staging of the abdomen and thorax. In a prospective clinical study the routine use of chest CT and bone scan did not provide additional information on metastases in HCC patients with BCLC $0, A, C$ or D stages and led to a stage shift from BCLC B to C in about 5\% of patients [29].

\section{Stage-Dependent Treatment}

The current European Guidelines endorse the BCLC algorithm for treatment decisions. The heterogeneity of patients with intermediate (BCLC B) HCC requires further subclassification to support treatment decisions [30]. Some clinical scenarios are not adequately reflected in the algorithm. Treatment decisions for the individual patient therefore should be reached by an interdisciplinary team. The BCLC treatment recommendations are summarized in table 3.

Liver resection should be considered for patients with well-preserved liver function and in the absence of portal hypertension or elevated bilirubin. If nodules in these patients do not exceed $3 \mathrm{~cm}$, radiofrequency ablation (RFA) is as effective as resection concerning overall survival. When addressing all patients with tumors up to $5 \mathrm{~cm}$, a large meta-analysis reported better survival for patients who had undergone hepatic resection [31]. Patients not suitable for resection should undergo local tumor ablation with RFA being the preferred recommended option. Percutaneous ethanol injection has been abandoned as a routine tool but still is an option in patients who are technically no candidates for RFA (e.g. expected heat-sink effect due to large vessels near the tumor). If the nodule planned for RFA is $3-5 \mathrm{~cm}$ in size, clinical studies support tumor embolization in advance of RFA [32, 33] which, however, is not part of the BCLC algorithm. Patients within the 'Milan criteria' are candidates for liver transplantation, but the current guidelines for the evaluation of these patients for transplantation need to be followed strictly. Because of the high drop-out rates on the waiting lists for transplantation, bridging therapy - transarterial chemoembolization (TACE), RFA or resection should be considered.

Patients in BCLC B benefit from locoregional transarterial therapy, and TACE is the treatment of choice, aiming at a combination of cytotoxic and ischemic effects. In the absence of internationally accepted treatment standards for TACE the comparison of results of (randomized controlled) trials is hampered due to a broad variety of applied techniques. In a prospective randomized controlled trial (RCT), TACE applying drug-eluting beads was as effective as chemoembolization using Lipiodol-Gelfoam particles, but had less systemic toxic side effects [34].

Sorafenib is the only systemic therapy for which prolonged overall survival of patients with advanced disease has been demonstrated, and it is therefore recommended in cases of well-preserved liver function (Child A) [3,14]. In patients with impaired liver function (Child B) there are no data from RCTs showing a survival benefit for these patients. At present, no second-line therapy can be recommended in case of tumor progression in patients treated with sorafenib, and the results of many ongoing clinical trials are eagerly awaited. 


\section{Future Developments}

The therapeutic armamentarium for HCC is evolving at a fast pace, with many therapeutic options currently being under evaluation. They include a variety of local or locoregional treatment modalities (e.g. Y90 radioembolization, cryoablation, microwave ablation and brachytherapy by external beam radiation or by interstitial high-dose brachytherapy). Furthermore the combination (in parallel or sequentially) of different locoregional treatments and the combination of systemic and locoregional treatment are being studied. New systemic treatment agents and their potential as second-line options or in combination with sorafenib in first-line settings are under evaluation.

Y90 radioembolization shows promising results in patients with intermediate-stage HCC, and a RCT randomizing against TACE needs to be conducted. So far, data from single-armed cohort studies and matched-pair evaluations of Y90 radioembolization report promising results and include patients with a high tumor load of the liver or portal vein thrombosis. TACE on the other hand is recommended because of two RCTs in patients with rather little tumor load of the liver [35-39]. In patients with a tumor load of the liver $>70 \%$ or in cases with significant elevation of bilirubin levels Y90 radioembolization is of no benefit. More insight will be obtained from RCTs evaluating Y90 radioembolization against systemic treatment with sorafenib or in combination with systemic treatment with sorafenib (SORAMIC). It may be expected that $\mathrm{Y} 90$ radioembolization will prolong survival in patients with more advanced BCLC B stages and eventually even in BCLC C stages. A RCT addressing the combination of TACE and sorafenib (SPACE) failed to achieve its primary endpoint. This is attributed mainly to problems in trial design and execution.

The elucidation of molecular mechanisms of hepatocarcinogenesis resulted in the development of numerous molecular-targeted therapies that need to be tested in clinical settings as to whether they are superior to sorafenib in prolongation of survival for patients with advanced HCC. The majority of targeted therapies is based on tyrosine kinase inhibition, amongst other targets mainly in angiogenetic pathways and/or the mTOR pathway.

In a phase II study on tivantinib, patients with high c-Met expression in the tumor tissue showed significant prolongation of time to progression and overall survival compared to placebo in patients who failed on sorafenib, while patients lacking this overexpression did not benefit. The substance is currently being evaluated in a phase III trial. The important information derived from this phase II trial was that identifying biomarkers from tumor tissue can be predictive for the effect of targeted therapies. This will lead the current trend of diagnosing HCC non-invasively towards more frequent tumor biopsies. Further treatment concepts currently studied are based on immunotherapy and oncolytic viruses [40].

Studies addressing the combination of a second systemic agent in combination with sorafenib are limited because of the significant toxicity when administering two anticancerous drugs to patients with liver function impairment because of liver cirrhosis. So far, no drug combination has been shown to be superior to monotherapy with sorafenib in patients with advanced HCC concerning survival.

\section{Conclusion}

The burden of HCC continues to increase worldwide, but effective management strategies have changed the picture to an optimistic one. New diagnostic criteria and staging algorithms help the managing physician, and a growing armamentarium of treatment options benefits the patients. Liver function frequently still represents a major limitation for the therapeutic options that can be offered. The most encouraging approach therefore remains 
prevention of HCC. Strategies include prevention and treatment of viral hepatitis and careful search for other causes of chronic liver disease. Optimal - and cost-effective - surveillance strategies need to be developed and followed.

\section{Disclosure Statement}

The authors declare no conflicts of interest. There was no funding.

\section{References}

1 Mittal S, El-Serag HB: Epidemiology of hepatocellular carcinoma: consider the population. J Clin Gastroenterol 2013;47(suppl):S2-S6.

2 Bruix J, Sherman M: Management of hepatocellular carcinoma: an update. Hepatology 2011;53:1020-1022.

-3 European Association for the Study of the Liver; European Organisation for Research and Treatment of Cancer: EASL-EORTC clinical practice guidelines: management of hepatocellular carcinoma. J Hepatol 2012; 56:908-943.

4 Song DS, Bae SH: Changes of guidelines diagnosing hepatocellular carcinoma during the last ten-year period. Clin Mol Hepatol 2012;18:258-267.

-5 Schütte K, Bornschein J, Malfertheiner P: Hepatocellular carcinoma - epidemiological trends and risk factors. Dig Dis 2009;27:80-92.

-6 Floriani I, D’Onofrio M, Rulli E, et al: Leistungsfähigkeit der bildgebenden Verfahren bei der Diagnose des hepatozellulären Karzinoms: Systematischer Review und Metaanalyse. Ultraschall Med 2013;34:454-462.

7 Omata M, Lesmana LA, Tateishi R, et al: Asian Pacific Association for the Study of the Liver consensus recommendations on hepatocellular carcinoma. Hepatol Int 2010;4:439-474.

-8 Hadziyannis E, Sialevris K, Georgiou A, Koskinas J: Analysis of serum $\alpha$-fetoprotein-L3\% and des- $\gamma$ carboxyprothrombin markers in cases with misleading hepatocellular carcinoma total $\alpha$-fetoprotein levels. Oncol Rep 2013;29:835-839.

-9 Ertle JM, Heider D, Wichert M, et al: A combination of $\alpha$-fetoprotein and des- $\gamma$-carboxy prothrombin is superior in detection of hepatocellular carcinoma. Digestion 2013;87:121-131.

$\rightarrow 10$ Joo I, Choi BI: New paradigm for management of hepatocellular carcinoma by imaging. Liver Cancer 2012;1: 94-109.

11 Niu Y, Huang T, Lian F, Li F: Contrast-enhanced ultrasonography for the diagnosis of small hepatocellular carcinoma: a meta-analysis and meta-regression analysis. Tumour Biol 2013;34:3667-3674.

12 Galassi M, Iavarone M, Rossi S, et al: Patterns of appearance and risk of misdiagnosis of intrahepatic cholangiocarcinoma in cirrhosis at contrast enhanced ultrasound. Liver Int 2013;33:771-779.

13 Barreiros AP, Piscaglia F, Dietrich CF: Contrast enhanced ultrasound for the diagnosis of hepatocellular carcinoma (HCC): comments on AASLD guidelines. J Hepatol 2012;57:930-932.

14 Greten TF, Malek NP, Schmidt S, et al: Diagnosis of and therapy for hepatocellular carcinoma. Z Gastroenterol 2013;51:1269-1326.

$\checkmark 15$ Granito A, Galassi M, Piscaglia F, et al: Impact of gadoxetic acid (Gd-EOB-DTPA)-enhanced magnetic resonance on the non-invasive diagnosis of small hepatocellular carcinoma: a prospective study. Aliment Pharmacol Ther 2013;37:355-363.

16 Kudo M, Matsui O, Sakamoto M, et al: Role of gadolinium-ethoxybenzyl-diethylenetriamine pentaacetic acidenhanced magnetic resonance imaging in the management of hepatocellular carcinoma: consensus at the Symposium of the 48th Annual Meeting of the Liver Cancer Study Group of Japan. Oncology 2013;84(suppl 1):21-27.

17 Murakami T, Imai Y, Okada M, et al: Ultrasonography, computed tomography and magnetic resonance imaging of hepatocellular carcinoma: toward improved treatment decisions. Oncology 2011;81(suppl 1):86-99.

18 Forner A, Vilana R, Ayuso C, et al: Diagnosis of hepatic nodules $20 \mathrm{~mm}$ or smaller in cirrhosis: prospective validation of the noninvasive diagnostic criteria for hepatocellular carcinoma. Hepatology 2008;47:97-104.

$\checkmark 19$ Verslype C, Rosmorduc O, Rougier P: Hepatocellular carcinoma: ESMO-ESDO Clinical Practice Guidelines for diagnosis, treatment and follow-up. Ann Oncol 2012;23(suppl 7):vii41-vii48.

20 Silva MA, Hegab B, Hyde C, et al: Needle track seeding following biopsy of liver lesions in the diagnosis of hepatocellular cancer: a systematic review and meta-analysis. Gut 2008;57:1592-1596.

21 Llovet JM, Brú C, Bruix J: Prognosis of hepatocellular carcinoma: the BCLC staging classification. Semin Liver Dis 1999;19:329-338.

22 Okusaka T, Okada S, Ueno H, et al: Satellite lesions in patients with small hepatocellular carcinoma with reference to clinicopathologic features. Cancer 2002;95:1931-1937.

23 Yuki K, Hirohashi S, Sakamoto M, et al: Growth and spread of hepatocellular carcinoma. A review of 240 consecutive autopsy cases. Cancer 1990;66:2174-2179. 
24 Senthilnathan S, Memon K, Lewandowski RJ, et al: Extrahepatic metastases occur in a minority of hepatocellular carcinoma patients treated with locoregional therapies: analyzing patterns of progression in 285 patients. Hepatology 2012;55:1432-1442.

25 Uchino K, Tateishi R, Shiina S, et al: Hepatocellular carcinoma with extrahepatic metastasis: clinical features and prognostic factors. Cancer 2011;117:4475-4483.

26 Sneag DB, Krajewski K, Giardino A, et al: Extrahepatic spread of hepatocellular carcinoma: spectrum of imaging findings. AJR Am J Roentgenol 2011;197:W658-W664.

27 Lee H: Management of patients with hepatocellular carcinoma and extrahepatic metastasis. Dig Dis 2011;29: 333-338.

28 Okuda K: Natural history of hepatocellular carcinoma including fibrolamellar and hepato-cholangiocarcinoma variants. J Gastroenterol Hepatol 2002;17:401-405.

29 Jin Y, Lee HC, Lee D, et al: Role of the routine use of chest computed tomography and bone scan in staging workup of hepatocellular carcinoma. J Hepatol 2012;56:1324-1329.

$\$ 30$ Bolondi L, Burroughs A, Dufour J, et al: Heterogeneity of patients with intermediate (BCLC B) hepatocellular carcinoma: proposal for a subclassification to facilitate treatment decisions. Semin Liver Dis 2012;32:348359.

31 Zhou Y, Zhao Y, Li B, et al: Meta-analysis of radiofrequency ablation versus hepatic resection for small hepatocellular carcinoma. BMC Gastroenterol 2010;10:78.

-32 Kim JH, Won HJ, Shin YM, et al: Medium-sized (3.1-5.0 cm) hepatocellular carcinoma: transarterial chemoembolization plus radiofrequency ablation versus radiofrequency ablation alone. Ann Surg Oncol 2011;18:16241629.

-33 Morimoto M, Numata K, Kondou M, et al: Midterm outcomes in patients with intermediate-sized hepatocellular carcinoma: a randomized controlled trial for determining the efficacy of radiofrequency ablation combined with transcatheter arterial chemoembolization. Cancer 2010;116:5452-5460.

34 Lammer J, Malagari K, Vogl T, et al: Prospective randomized study of doxorubicin-eluting-bead embolization in the treatment of hepatocellular carcinoma: results of the PRECISION V study. Cardiovasc Intervent Radiol 2010;33:41-52.

35 Lo C, Ngan H, Tso W, et al: Randomized controlled trial of transarterial lipiodol chemoembolization for unresectable hepatocellular carcinoma. Hepatology 2002;35:1164-1171.

-36 Llovet JM, Real MI, Montaña X, et al: Arterial embolisation or chemoembolisation versus symptomatic treatment in patients with unresectable hepatocellular carcinoma: a randomised controlled trial. Lancet 2002;359:1734-1739.

37 Salem R, Lewandowski RJ, Kulik L, et al: Radioembolization results in longer time-to-progression and reduced toxicity compared with chemoembolization in patients with hepatocellular carcinoma. Gastroenterology 2011;140:497-507.e2.

-38 Salem R, Lewandowski RJ, Mulcahy MF, etal: Radioembolization for hepatocellular carcinoma using Yttrium-90 microspheres: a comprehensive report of long-term outcomes. Gastroenterology 2010;138:52-64.

39 Sangro B, Carpanese L, Cianni R, et al: Survival after yttrium-90 resin microsphere radioembolization of hepatocellular carcinoma across Barcelona clinic liver cancer stages: a European evaluation. Hepatology 2011;54: 868-878.

40 Heo J, Reid T, Ruo L, et al: Randomized dose-finding clinical trial of oncolytic immunotherapeutic vaccinia JX-594 in liver cancer. Nat Med 2013;19:329-336.

41 Yu NC, Chaudhari V, Raman SS, et al: CT and MRI improve detection of hepatocellular carcinoma, compared with ultrasound alone, in patients with cirrhosis. Clin Gastroenterol Hepatol 2011;9:161-167.

-42 Khalili K, Kim TK, Jang H, et al: Optimization of imaging diagnosis of 1-2 cm hepatocellular carcinoma: an analysis of diagnostic performance and resource utilization. J Hepatol 2011;54:723-728.

43 Seitz K, Bernatik T, Strobel D, et al: Contrast-enhanced ultrasound (CEUS) for the characterization of focal liver lesions in clinical practice (DEGUM Multicenter Trial): CEUS vs. MRI - a prospective comparison in 269 patients. Ultraschall Med 2010;31:492-499.

44 Seitz K, Strobel D, Bernatik T, et al: Contrast-enhanced ultrasound (CEUS) for the characterization of focal liver lesions - prospective comparison in clinical practice: CEUS vs. CT (DEGUM multicenter trial). Parts of this manuscript were presented at the Ultrasound Dreiländertreffen 2008, Davos. Ultraschall Med 2009;30:383389.

45 Jang H, Kim TK, Wilson SR: Small nodules (1-2 cm) in liver cirrhosis: characterization with contrast-enhanced ultrasound. Eur J Radiol 2009; 72:418-424.

46 Baek C, Choi J, Kim K, et al: Hepatocellular carcinoma in patients with chronic liver disease: a comparison of gadoxetic acid-enhanced MRI and multiphasic MDCT. Clin Radiol 2012;67:148-156.

47 Sangiovanni A, Manini MA, Iavarone M, et al: The diagnostic and economic impact of contrast imaging techniques in the diagnosis of small hepatocellular carcinoma in cirrhosis. Gut 2010;59:638-644.

48 Bashir MR, Gupta RT, Davenport MS, et al: Hepatocellular carcinoma in a North American population: does hepatobiliary MR imaging with Gd-EOB-DTPA improve sensitivity and confidence for diagnosis? J Magn Reson Imaging 2013;37:398-406. 